\title{
The Experimental Effect of Parental Attentiveness on Children's Physical Activity Behavior
}

\author{
Michael J. Rebold, Ph.D., CSCS ${ }^{*}$, Mallory S. Kobak, Ph.D., CSCS ${ }^{1}$, Cody A. Croall M.S. ${ }^{2}$, Emily A. \\ Cumberledge M.S. ${ }^{2}$, Melanie Hall M.S. ${ }^{2}$, Lindsey Raumikaitis M.S. ${ }^{2}$ \\ ${ }^{1}$ Department of Integrative Exercise Science, Hiram College, Hiram, USA \\ ${ }^{2}$ Department of Exercise Science, Bloomsburg University of Pennsylvania, Bloomsburg, USA
}

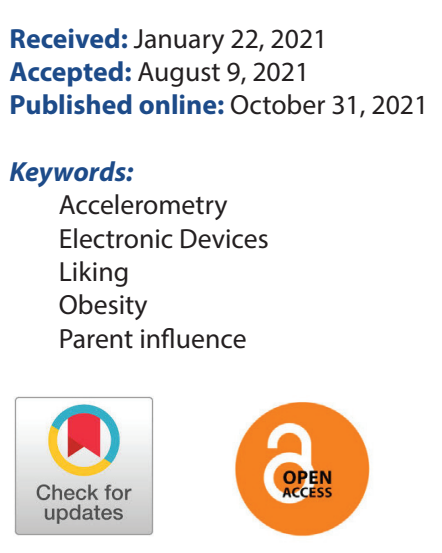

\begin{abstract}
OBJECTIVES To assess children's physical and sedentary activity behavior, enjoyment (i.e., liking), and preference during two separate experimental conditions: parent attentive and parent non-attentive.

METHODS Ten children ( $n=6$ boys, 4 girls), 3 to 6 years old, along with one parent ( $n=6$ mothers, 4 fathers) per child participated in each condition on separate days for 30-minutes in which they could choose from a variety of physical and/or sedentary activities.

RESULTS A greater number of accelerometer counts $(p=0.04)$ were accumulated during the parent attentive $(96,547 \pm 33,075.26$ counts) condition than the parent non-attentive $(48,316.30 \pm 46,101.47$ counts) condition. More time $(p=0.007)$ was allocated to sedentary activities during the parent non-attentive (19.5 \pm 13.3 minutes) condition than parent attentive ( $2.8 \pm 3.55$ minutes) condition. Children liked $(p=0.004)$ the parent attentive $(9.05 \pm 1.21 \mathrm{~cm})$ condition more than parent non-attentive $(4.42 \pm 3.18 \mathrm{~cm})$ condition. A non-significant $(p=0.21)$ proportion of children identified the parent attentive $(70 \%)$ as their preferred condition over the parent non-attentive condition.

CONCLUSIONS Parental attentiveness may be an important component to take into consideration when trying to maximize children's physical activity behavior and enjoyment while simultaneously reducing sedentary behavior.
\end{abstract}

(c) The Asian Society of Kinesiology and the Korean Academy of Kinesiology

\section{Introduction}

Childhood obesity continues to be a serious medical condition in the United States resulting in children and adolescents being at an increased risk for the development of many obesity-related disorders such as diabetes, high blood pressure, and high cholesterol [1-3]. According to the Centers for Disease Control (CDC) and Prevention [4], 13.9\% of 2- to 5 -year olds are obese, $18.4 \%$ of 6 - to 11 -year olds, and $20.6 \%$ 12- to 19-year olds. Due to this continued increase in the

*Correspondence: Michael J. Rebold. Department of Integrative Exercise Science, Hiram College, Hiram, USA; Phone: (330) 569-5365; E-mail: reboldmj@hiram.edu prevalence of childhood obesity, it remains imperative that efforts are being placed into the development of physical activity interventions to decrease the number of obesityrelated disorder cases. According to the CDC [4], one of the best strategies to reduce childhood obesity is to improve the exercise habits of the entire family. This type of strategy has been examined previously in several experimental and nonexperimental investigations [5-9]. Since it is important to influence physical activity behaviors at an early age to decrease the risk of childhood obesity and obesity-related disorders, it is necessary to direct our focus on the parental interaction that occurs during children's physical activity interventions. This 
is imperative, because parent(s) are considered the primary role model for younger children and can therefore, influence physical activity behaviors to the greatest extent [5-16].

It has been recognized that parents play an influential role in shaping children's physical activity participation through support and behavioral modeling [7,14]. The Social Learning Theory can be used to explain this relationship that exists between parents and their children's habits and attitudes towards physical activity $[11,15]$. This theory suggests that if parents model physical activity (i.e., exercise in front of their children), then their children are more likely to be active. Another explanation for how parents may influence their children's physical activity is provided by the Behavioral Choice Theory. Children's decision to participate in one behavior (e.g., physical activity) is weighted against the decision to participate in an alternative behavior (e.g., sedentary activity) [16]. This theory suggests that parents must encourage physical activity rather than sedentary activity. This can be facilitated by parents creating environments that promote enjoyment of physical activity.

Recent investigations have attempted to understand the casual impact parental interactions has on children's physical activity [5,6]. Rebold and colleagues [5] conducted an experimental study which investigated how parents playing and parents watching influenced their children's physical activity behaviors. It was found that children were more physically active when their parents participated $(109,523 \pm$ 32,155 accelerometer counts) in physical activity with them as compared to watching $(85,624 \pm 44,985$ accelerometer counts) them play or allowing them play alone $(67,938 \pm 37,857$ accelerometer counts) [5]. One of the limitations associated with this study was that the conditions were conducted inside a gymnasium and did not completely resemble a natural and realistic environment for children. To address this limitation Kobak and colleagues [6] conducted an experimental study that investigated children's physical activity while playing with their parents and their friends on an outdoor playground. It was found that children were more physically active when they played with their friends $(93,363 \pm 22,608$ accelerometer counts) as compared to playing with their parents $(87,503$ $\pm 37,063$ accelerometer counts) or playing alone $(70,672 \pm$
35,228 accelerometer counts) [6]. It has also been recognized that parents play a particularly important role in their children's physical activity behaviors when they are younger (e.g., less than six years of age) [12]. Kobak and colleagues' [6] investigation focused on $8.2 \pm 1.7$ years old children while Rebold and colleagues' [5] study focused on $4.05 \pm 0.9$ years old children, which may partially explain why parents had a greater impact on physical activity in Rebold and colleagues' [5] study. This does circle back to the CDC recommendations on why it is important to improve the exercise habits of the entire family, because different family members will have differing effects on children's physical activity behaviors at different life stages (e.g., parents at younger ages; siblings at older ages).

An environment that parents often take their children to play at is the playground, and when parents are at the playground many of them sit on the benches and engage in sedentary behaviors (e.g., work on their laptop, scroll through apps on their cell phone, etc.). But, can the attentiveness levels (attentive vs. non-attentive) of parents while watching their children play actually be influencing how much physical activity their children are actually engaging in? The purpose of this study was to assess the amount, intensity, enjoyment (i.e., liking), and preference of children's physical activity in a controlled setting under two experimental, conditions: parent attentive and parent non-attentive. To the best of our knowledge, this was the first study to experimentally examine the effects of parental attentiveness on children's physical activity behavior. This study involved the manipulation of what parents were required to do (attentive and non-attentive) while sitting on a bench watching their children interact in an environment that included both sedentary and physical activity options. It was hypothesized that the parent attentive condition would increase children's physical activity behavior relative to the parent non-attentive condition.

\section{Methods}

\section{Subjects}

Ten children ( $n=6$ boys, 4 girls) between the ages of 3-6 years old (mean age $=4.75 \pm 1.28$ years) $<$ Table $1>$, along with one parent ( $n=6$ mothers, 4 fathers) per child were 
recruited from the local community through flyers. Children, along with one parent per child participated in two conditions (parent attentive and parent non-attentive) on separate days in a 4,360 square foot gymnasium. Both conditions took place inside a gymnasium even though this was a noted limitation in previous studies $[5,6]$. Parents of the children indicated to the researchers that they preferred the gymnasium over the playground due to driving distance, and it was also easier to reserve the gymnasium to ensure no other children and parents were present that could have influenced our data collection procedures and our findings. Children were excluded from the study if they had any contraindications to exercise (i.e., orthopedic injury, cardiovascular disorder, etc.). This study was a within-subjects design with all children being exposed to the two conditions in a randomized order. The same parent participated in both the parent attentive and the parent non-attentive conditions with their child. This study received approval from the University Institutional Review Board.

Table 1. Children's Physical Characteristics (Data are Means $\pm S D$ )

\begin{tabular}{lll}
\hline & Boys $(\boldsymbol{n}=\mathbf{6})$ & Girls $(\boldsymbol{n}=\mathbf{4})$ \\
\hline Age (yrs) & $5 \pm 1.26$ & $4.5 \pm 1.29$ \\
$p=0.59$ & $114.08 \pm 13.14$ & $110.78 \pm 10.77$ \\
Height $(\mathrm{cm})$ & \\
$p=0.67$ & $21.41 \pm 3.57$ & $19.89 \pm 3.31$ \\
Weight $(\mathrm{kg})$ & \\
$p=0.51$ & \\
\hline
\end{tabular}

\section{Procedures}

Upon arrival for the first visit, parental consent and child assent was obtained. The child then underwent anthropometric assessments (i.e., height and weight) $<$ Table $1>$. Children were measured for height via a stadiometer and weight by using a balance beam scale (Health O Meter, Chicago, IL). In addition, gender and age were also recorded. During each of the two separate conditions, participants were taken to a 4,360 square foot gymnasium where there was a variety of age-appropriate physical activity options and sedentary alternatives. The set-up of the gymnasium was similar to what was utilized in previous studies conducted by Rebold colleagues [5], Sanders and colleagues [17], and Barkley and colleagues [18]. Physical activity equipment consisted of jump ropes, basketballs and hoops, hurdles, footballs, soccer balls, and multiple obstacle courses which was made up of gymnastic soft-play equipment $[5,17,18]$. The sedentary activity area was equipped with one table and one chair to accommodate the child. Children were instructed that they had to sit if they were playing with the sedentary alternatives. The age-appropriate sedentary alternatives included action figures, toy dolls, board games, reading books, crayons, markers, colored pencils, blank paper for drawing, and coloring sheets $[5,17,18]$. After being shown the physical activity options and sedentary alternatives the child was fitted with a validated accelerometer (Actigraph GT1M, Pensacola, FL) that was comfortably wrapped around their waist and it recorded the number of physical activity counts per session for each child [19]. After being fitted with the accelerometer, the child was given permission to participate in any of the physical and/or sedentary activities they wished, in any pattern for 30-minutes by themselves (i.e., no other children present) while their parent was seated in a chair in the gymnasium.

The parent non-attentive condition consisted of the child participating in the physical and/or sedentary activities while their parent was seated in a chair in the gymnasium. During this condition, the parent was instructed not to speak with their child and to remain active on their electronic device (e.g., laptop or mobile tablet) for the entirety of the condition. Parents were not allowed to leave their chair, and if their child tried to talk to them, parents were instructed to tell their child that they were busy. The parent attentive condition was the same as the parent non-attentive condition except the parent was allowed to speak with their child, however, they were informed not to instruct the child about which activities they should or should not utilize. Parents were not allowed to leave their chair, and while seated in the chair parents were not allowed to be on their electronic devices. Apart from the participant child, the parent, and research personnel, there were no other individuals present in the gymnasium during each condition.

In addition to accelerometry data, the time children allocated to physical and sedentary activities during each 
30-minute activity session was recorded by direct observation of research personnel using a stopwatch. To record how much time children spent engaged in the physical activities, the research personnel started the stopwatch when children were actively moving (i.e., walking, running, jumping, etc.) and then stopped the stopwatch as soon as they stopped moving or sat down. And, to record how much time children spent engaged in the sedentary activities, the research personnel started the stopwatch when children were sitting in the chair located by the table with the sedentary alternatives and stopped the stopwatch as soon as they stood up and started to move. As stated previously, children were instructed to remain seated while engaged in the sedentary alternatives. At the end of each 30-minute session, each child was asked to indicate their liking of the activity using a validated visual analog scale by marking on a 10-cm line anchored by "like it very much" on the left and "do not like it at all" on the right [20]. Children were then asked if they would like to play for an additional 10-minutes or if they wished to be finished for the session. If a child elected to participate in an additional 10-minutes of activity they followed identical procedures to their initial 30-minutes for that day. After both conditions were completed, each child was asked to indicate which condition (parent attentive or parent non-attentive) was their favorite.

\section{Analytic plan}

All data were analyzed with SPSS version 20.0 (SPSS Incorporated, Chicago, IL) with an a-priori a level of $\leq 0.05$. Independent samples t-tests were used to examine any sex differences (boys, girls) in physical characteristics (i.e., age, height, weight). A two sex (boys, girls) by two condition (parent attentive, parent non-attentive) analysis of co-variance (ANCOVA) with repeated measures on social condition and co-varying for age of the child (3-6 years old) and sex of the parent (mother, father) was utilized to assess differences in accelerometer counts and sedentary minutes during the 30-minute session. Post-hoc analyses for all significant main and interaction effects were completed by using t-tests with the Benjamini and Hochberg False Discovery Rate correction [21]. A Wilcoxon signed rank test was used to examine any differences in the children's decision to choose to participate in the additional 10-minutes across the two conditions. Additionally, a chi-square analysis was performed to assess any differences in the children's preference of the two conditions. Finally, a Pearson's correlation coefficient was performed to assess the relationship between accelerometer counts and sedentary minutes of the two conditions. Differences in estimated METs and kilocalories across the two social conditions were not analyzed as each of these variables was ultimately calculated from accelerometer counts. Therefore, analyzing estimated METs and kilocalories using the above ANCOVA model would yield the exact same results as the analysis of accelerometer counts.

\section{Results}

Independent samples t-tests revealed no significant differences < Table $1>$ between boys and girls in any of the physical characteristics (age, height, and weight).

\section{Social conditions}

Accelerometer counts

A Pearson's chi-squared test for normality revealed that accelerometer counts were normally distributed for both the parent attentive and parent non-attentive conditions $\left(x^{2}[9, N=\right.$ $10]=\geq 16.93, p \leq 0.05)$. There was a significant main effect of condition for accelerometer counts $(\mathrm{F}=5.79, p=0.04)<$ Table $2>$. More counts $(\mathrm{t}=2.41, p=0.04)$ were accumulated during the parent attentive $(96,547 \pm 33,075.26$ counts) condition than the parent non-attentive $(48,316.30 \pm 46,101.47$ counts) condition. There were no significant main effect for sex or interaction effect between sex and condition $(\mathrm{F}=1.04, p=$ $0.34)$.

\section{Time allocated to sedentary activities}

A Pearson's chi-squared test for normality revealed that sedentary minutes were normally distributed for both the parent attentive and parent non-attentive conditions $\left(x^{2}[9\right.$, $N=10]=\geq 17.21, p \leq 0.05)$. There was a significant main effect of condition for time allocated to sedentary activities $(\mathrm{F}=11.99, p=0.007)<$ Table $2>$. More time $(\mathrm{t}=-3.46, p=$ 0.007 ) was allocated to sedentary activities during the parent 
Table 2. Children's Accelerometer Counts, Sedentary Minutes, Liking, Bonus Activity, and Preference of Condition (Data are Means \pm SD)

\begin{tabular}{|c|c|c|}
\hline & $\begin{array}{l}\text { Parent Attentive } \\
(n=10)\end{array}$ & $\begin{array}{l}\text { Parent Non- } \\
\text { Attentive }(n=10)\end{array}$ \\
\hline $\begin{array}{l}\text { Accelerometer counts } \\
\text { (counts) }\end{array}$ & $96,547 \pm 33,075.26^{*}$ & $48,316.30 \pm 46,101.47$ \\
\hline \multicolumn{3}{|l|}{$\eta^{2}=0.12$} \\
\hline \multicolumn{3}{|l|}{$p=0.04$} \\
\hline $\begin{array}{l}\text { Sedentary minutes } \\
\text { (min) }\end{array}$ & $2.8 \pm 3.55$ & $19.5 \pm 13.3 \dagger$ \\
\hline \multicolumn{3}{|l|}{$\eta^{2}=0.14$} \\
\hline \multicolumn{3}{|l|}{$p=0.007$} \\
\hline Liking (cm) & $9.05 \pm 1.21^{*}$ & $4.42 \pm 3.18$ \\
\hline \multicolumn{3}{|l|}{$\eta^{2}=0.14$} \\
\hline \multicolumn{3}{|l|}{$p=0.004$} \\
\hline $\begin{array}{l}\text { Bonus Activity } \\
\text { (percent) }\end{array}$ & $60 \%$ & $40 \%$ \\
\hline \multicolumn{3}{|l|}{$p=0.56$} \\
\hline $\begin{array}{l}\text { Preference of } \\
\text { condition (number) }\end{array}$ & 7 & 3 \\
\hline \multicolumn{3}{|l|}{$p=0.21$} \\
\hline \multicolumn{3}{|c|}{$\begin{array}{l}\text { Data are Mean } \pm \text { SEM } \\
{ }^{*} \text { condition was significantly greater than parent non-attentive } \\
\text { tcondition was significantly greater than parent attentive } \\
p<0.05 \text { for all }\end{array}$} \\
\hline
\end{tabular}

non-attentive $(19.50 \pm 13.30$ minutes $)$ condition than the parent attentive $(2.80 \pm 3.55$ minutes $)$ condition. There were no significant main effect for sex or interaction effect between sex and condition $(\mathrm{F}=0.002, p=0.97)$.

\section{Liking for each condition}

There was a significant main effect of condition for liking $(\mathrm{F}=15.18, p=0.004)<$ Table $2>$. Children liked $(\mathrm{t}=3.90, p$ $=0.004)$ the parent attentive $(9.05 \pm 1.21 \mathrm{~cm})$ condition more than the parent non-attentive $(4.42 \pm 3.18 \mathrm{~cm})$ condition. There were no significant main effect for sex or interaction effect between sex and condition $(\mathrm{F}=0.06, p=0.82)$.

\section{Bonus period of activity}

There was not a significant difference $(Z=-0.58, p=0.56)$ $<$ Table $2>$ between the proportion of children who chose to participate in the additional 10-minute bonus period between the parent attentive $(n=6)$ and parent non-attentive $(n=4)$ conditions.
Preference of condition

Chi-square analysis revealed a non-significant difference $\left(x^{2}=1.60, p=0.21\right)<$ Table $2>$ between the number of children that indicated they preferred the parent attentive $(n=7)$ condition versus the parent non-attentive $(n=3)$ condition.

\section{Relationship between accelerometer counts and sedentary minutes}

A Pearson correlation revealed a moderate negative relationship ( $r=-0.31)$ between accelerometer counts and sedentary minutes for the parent attentive condition. For the parent non-attentive condition, there was almost a perfect strong negative relationship $(r=-0.94)$. This suggests that as children engaged in more physical activity their time spent in sedentary alternatives decreased.

\section{Discussion}

To the best of our knowledge this was the first experimental study examining the effects of parental attentiveness while watching their child play and how it affects their child's physical activity behavior. The results of this current study indicated that children were more physically active when parents were attentive versus when parents were non-attentive. Children had a 99\% increase in accelerometer counts from the parent non-attentive condition to the parent attentive condition. Children also had a 596\% decrease in time spent in sedentary activity from the parent non-attentive condition to the parent attentive condition. Finally, children reported a $105 \%$ increase in liking from the parent non-attentive condition to the parent attentive condition, which may explain why majority of the children (70\%) indicated that they preferred the parent attentive condition. Additionally, only $60 \%$ of children elected to participate in the 10-minute bonus activity when their parent was attentive versus when their parent was non-attentive. This finding was surprising because previous studies that have implemented similar protocols have found that when children like (i.e., enjoy) a condition more, they are more likely to engage in the 10-minute bonus activity for that condition $[5,6]$. These current findings indicate that children are likely to engage in more physical activity and have less sedentary minutes when parents are attentive. Overall, if 
parents are attentive while watching their children play, then that could lead to children participating in greater amounts of physical activity resulting in greater caloric expenditure and ultimately helping combat childhood obesity and obesityrelated disorders.

When converting accelerometer counts to caloric expenditure, children "burned" an average of 86.16 kcals in the 30-minute parent attentive condition and $40.83 \mathrm{kcals}$ in the parent non-attentive condition. The Freedson and colleagues [22] equation $\left(0.00094 \mathrm{x} \mathrm{cnts} \cdot \mathrm{min}^{-1}\right)+(0.1346 \mathrm{BW})$ - 7.37418 was utilized to convert accelerometer counts to caloric expenditure. Assuming children participated in similar 30-minute activity sessions three times per week for one year while using the caloric expenditure rates noted above, they would expend 10,483 and 4,968 kcals per year in the parent attentive and parent non-attentive conditions, respectively. This results in 5,515 kcals greater expenditure in the parent attentive than the parent non-attentive condition. Assuming 3,500 kcals expended is equivalent to one pound of body weight [3], this greater caloric expenditure when a parent is attentive would equate to 1.58 pounds of body weight lost per year more than the parent non-attentive condition. Additionally, children engaged in $111 \%$ more moderateto-vigorous physical activity (MVPA) from the parent nonattentive to the parent attentive condition. Cauwenberghe and colleagues [23], developed accelerometer cut points to classify physical activities by intensity in pre-school children (5.8 \pm 0.4 years). According to Cauwenberghe and colleagues [23], $\geq$ 2,340 counts/ $60 \mathrm{~s}$ would classify as MVPA. If we convert the 2,340 counts/ 60 s to 30 -minutes that would equate to 70,200 counts needed in 30-minutes to be considered engaging in MVPA. Children had 96,547 and 48,316.30 counts during the parent attentive and parent non-attentive conditions, respectively. Therefore, making simple changes, such as parents being attentive while their children are physical activity, may help offset this gradual excess weight gain.

As we hypothesized children accumulated significantly more accelerometer counts and less sedentary minutes when their parent was attentive versus the parent non-attentive condition. This is an important finding because parents simply being attentive offers a simple, yet effective means to increase physical activity in children. Rebold and colleagues [5] previously thought that children's physical activity would not significantly increase while parents were watching them play because parents were not modeling nor encouraging physical activity behavior, however, the findings from this current study demonstrate this not to be true. Therefore, the findings from this current study are not in agreement with the Social Learning and Behavioral Choice Theories, which suggests that parents play an important role in shaping children's physical activity participation through support and behavioral modeling $[7,14,16]$. Although not recorded, it appears from these findings that no matter how much parents modeled or encouraged physical activity to their children prior to participating in this current study, as soon as parents were non-attentive children significantly increased their sedentary activity. This possibly means that if a child receives no modeling nor encouragement from their parent(s), something as simple as the parent(s) being attentive while at the playground can result in significantly more physical activity. Our results provide casual evidence that when parents are attentive, it results in a positive impact on children's physical activity behavior.

The present results also demonstrated that children liked (i.e., enjoyed) the parent attentive condition more than the parent non-attentive condition with seven children reporting that the parent attentive condition was most preferred. As mentioned earlier, the Behavioral Choice Theory states that parents play a critical role in shaping children's interest and involvement in physical activity by creating environments that promote enjoyment of physical activity $[7,14,16,24]$. The findings from this current study are in agreement with the Behavioral Choice Theory, demonstrating something as simple as being parents being attentive has the potential to create a more enjoyable environment that promotes greater physical activity participation. However, what was surprising was that the parent attentive condition did not result in greater amounts of children to elect to participate in the additional 10-minute bonus period when compared to the parent non-attentive condition. Previous studies [2530] have suggested that if children find an activity to be more enjoyable it has the potential to lead to more engagement 
with the activity. A method that was previously utilized by Barkley and colleagues [30] was used in the present study to assess children's motivation by providing them with the option to play for an additional 10-minute bonus period after completing each initial 30-minute activity session. Currently, only $60 \%$ of children chose to participate in the additional 10-minute bonus period during the parent attentive condition while only $40 \%$ chose the bonus period in the parent nonattentive condition. It is reasonable to suggest that since more accelerometer counts were accumulated during the parent attentive condition and children liked and preferred this condition more, it would be expected that children would engage in more total physical activity if the session were not stopped at 30-minutes. Future studies should consider examining the impact of parental attentiveness on children's physical activity over a longer time frame such as the recommendation of 60-minutes set forth by the American College of Sports Medicine [3].

While this was the first experimental study to examine parental attentiveness on children's physical activity behavior and the results are encouraging, it is not without limitations. This study was conducted inside a gymnasium that utilized equipment (e.g., jump ropes, basketballs and hoops, footballs, soccer balls, obstacle courses made up of gymnastic soft-play equipment, etc.) that children may not necessarily have access to. Although the set-up of the gymnasium was similar to what has been utilized in previous studies $[5,17,18]$ the findings would possibly be more meaningful if children were allowed to be in a more natural and realistic environment similar to what Kobak and colleagues implemented while allowing children to play on a playground [6]. Children also participated in the conditions with only one parent, with 6 of the 10 parents being mothers. Although this is a realistic social environment at times because it has been reported that children generally tend to spend more time with their mothers than fathers [31], children do encounter interactions that involve the other parent and/or both parents simultaneously. It is possible that the presence of the other parent and/or both parents may differentially alter physical activity behavior. Another limitation is the 30 -minute free choice activity session. Since more accelerometer counts were accumulated, and children liked and preferred the parent attentive condition more, would children exhibit greater increases in total physical activity if the session were not capped at 30-minutes? A fourth limitation was that parental physical activity behavior was not assessed. Previous non-experimental studies have found that parents who are more physically active in front of their children or who encourage physical activity have children who are more physically active $[7-9,11,12]$. A fifth limitation was that the Freedson and colleagues [22] equation was utilized to convert accelerometer counts to caloric expenditure. This equation was developed based on the adult population, so it should be used with caution. It is recommended that the further development of equations applicable to children in free-living scenarios is needed. Lastly, we can only draw conclusions about the effect parental attentiveness has on children's physical activity behavior. We cannot make any inferences about the effect interactions with other adults may have. Children do interact with other adults in physically active environments such as teachers, sport coaches, older siblings, and extended family and these individuals may also effect physical activity behavior in children. Future studies should consider examining the following: the impact that both parents would have on children's physical activity behavior; the impact of parental attentiveness on children's physical activity over a longer time frame such as 60-minutes; comparing children whose parents are more physically active versus parents who are less physically active; and how other important adult figures (e.g., sport coaches, teachers) attentiveness may impact children's physical activity behavior.

\section{Conclusion}

This was the first experimental study designed to assess the impact of parental attentiveness on an acute bout of children's physical activity behavior. Prior experimental and non-experimental studies have suggested that when children are young, parents may have a significant impact on their physical activity behavior [5-9]. Since parental attentiveness was manipulated in the present study and its effects on children's physical activity behavior were assessed objectively we can conclude that when parents were attentive there was 
an increase in children's physical activity behavior, their liking of the activity, and decreased sedentary behavior relative to when parents were non-attentive. It is important for parents to create social and physical environments that support physical activity, so that children can make healthy choices and establish healthy behaviors. Based upon the present results, it is reasonable to conclude that parents should remain attentive in physical activity environments (e.g., playgrounds) to maximize physical activity behavior in children.

\section{Conflicts of Interest}

The researchers claim no conflicts of interest.

\section{References}

1. Hills AP, Andersen LB, Byrne NM. Physical activity and obesity in children. Br J Sports Med. 2012; 45:866-870.

2. Pradinuk M, Chanoine JP, Goldman RD. Obesity and physical activity in children. Can Fam Physician. 2011; 57:779-782.

3. Riebe D. ACSMs Guidelines for Exercise Testing and Prescription (10 ${ }^{\text {th }}$ ed.). Philadelphia, PA: Wolters Kluwer; 2018.

4. Hales CM, Carroll MD, Fryar CD, Ogden CL. Prevalence of obesity among adults and youth: United States, 2015-2016. NCHS data brief, no 288. Hyattsville, MD: National Center for Health Statistics; 2017.

5. Rebold MJ, Lepp A, Kobak MS, McDaniel J, Barkley JE. The effect of parental involvement on children's physical activity. J Pediatr. 2016; 170:206-210.

6. Kobak MS, Lepp A, Rebold MJ, Glickman E, Barkley JE. The experimental effect of parent versus peer on children's physical activity and sedentary behavior. Pediatr Exerc Sci. 2021; 1-7.

7. Beets MW, Cardinal BJ, Alderman BL. Parental social support and the physical activity-related behaviors of youth: a review. Heath Educ Behav. 2010; 37:621-644.

8. Dunton GF, Liao Y, Almanza E, Jerrett M, Spruijt-Metz D, Chih-Ping C. Joint physical activity and sedentary behavior in parent-child pairs. Med Sci Sports Exerc.
2012; 1473-1480.

9. Prochaska JJ, Rodgers MW, Sallis JF. Association of parent and peer support with adolescent physical activity. Res Q Exerc Sport. 2002; 73:206-210.

10. Trost SG, Sallis JF, Pate RR., Freedson PS, Taylor WC, Dowda M. Evaluating a model of parental influence on youth physical activity. Am J Prev Med. 2003; 25:277282.

11. Zecevic CA, Tremblay L, Lovsin T, Michel L. Parental influence on young children's physical activity. Int J Pediatr. 2011; 2010:468-526.

12. Trost SG, Loprinzi PD. Parental influences on physical activity behavior in children and adolescents: a brief review. Am J Lifestyle Med. 2011; 5:171-181.

13. Sallis JF, Prochaska JJ, Taylor WC. A review of correlates of physical activity of children and adolescents. Med Sci Sports Exerc. 2000; 32:963-975.

14. Ormrod JE. Human Learning ( $3^{\text {rd }}$ ed). Upper Saddle River, NJ: Prentice-Hall; 1999.

15. Bandura A. Self-efficacy: toward a unifying theory of behavioral change. Psychol Rev. 1977; 84:191-215.

16. Epstein LH. Integrating theoretical approaches to promote physical activity. Am J Prev Med. 1998; 15:257-265.

17. Sanders GJ, Peacock CA, Williamson ML, Wilson K, Carnes A, Barkley JE. The effect of friendship groups on children's physical activity: an experimental study. J Behav Health. 2014; 3:95-100.

18. Barkley JE, Salvy SJ, Sanders GJ, Dey S, Von Carlowitz KP, Williamson ML. Peer influence and physical activity behavior in young children: an experimental study. J Phys Act Health. 2014; 11:404-409.

19. Trost SG, Ward DS, Moorehead SM, Watson PD, Riner W, Burke JR. Validity of the computer sciences and applications (CSA) activity monitor in children. Med Sci Sports Exerc. 1998; 30:629-633.

20. Roemmich JN, Barkley JE, Lobarinas CL, Foster JH, White TM, Epstein LH. Association of liking and reinforcing value with children's physical activity. Physiol Behav. 2008; 93:1011-1018.

21. Benjamini Y, Hochberg Y. Controlling the false discovery rate: a practical and powerful approach to multiple 
testing. J R Stat Soc Series B. 1995; 57:289-300.

22. Freedson PS, Melanson E, Sirard J. Calibration of the computer science and applications, Inc. accelerometer. Med Sci Sports Exerc.1998; 30 (5): 777-781.

23. Cauwenberghe EV, LAbarque V, Trost SG, Bourdeaudhuij ID, Cardon G. Calibration and comparison of accelerometer cut points in preschool children. Int $\mathrm{J}$ Pediatr Obes. 2010; 6 (2-2):e582-589.

24. Veitch J, Salmon J, Ball K. Individual, social, and physical environmental correlates of children's active free-play: a cross-sectional study. Int J Behav Nut Phys Act. 2010; $7: 11$.

25. Beets MW, Pitetti KH, Forlaw L. The role of self-efficacy and referent specific social support in promoting rural adolescent girls' physical activity. Am J Health Behav. 2007; 31:227-237.

26. Epstein LH, Roemmich JN, Cavanaugh MD, Paluch RA. The motivation to be sedentary predicts weight change when sedentary behaviors are reduced. Int J Behav Nut Phys Act. 2011; 8:13.
27. Jago R, Macdonald-Wallis K, Thompson JL, Page AS, Brockman R, Fox KR. Better with a buddy: influence of best friends on children's physical activity. Med Sci Sports Exerc. 2010; 43:259-265.

28. Sallis JF, Alcaraz JE, McKenzie TL, Hovell MF. Predictors of change in children's physical activity over 20 months. Variations by gender and level of adiposity. Am J Prev Med. 1999; 16:222-229.

29. Salvy SJ, Roemmich JN, Bowker JC, Romero ND, Stadler PJ, Epstein LH. Effect of peers and friends on youth physical activity and motivation to be physically active. J Pediatr Psychol. 2009; 34:217-225.

30. Barkley JE, Salvy SJ, Sanders GJ, Williamson M, Dey S, Von Carlowitz, K. Peer influence and physical activity in young children: a controlled study. Med Sci Sports Exerc. 2011; 43:S224.

31. Halle T. Charting parenthood: a statistical portrait of fathers and mothers in America. Child Trends; 2006. Available from: http://fatherhood.hhs.gov/charting02. (Accessed July 10, 2020) 